\title{
环糊精参与的过渡金属催化有机反应
}

\author{
陈雅琪桂金鐏斌朱丽君项玉芝夏道宏* \\ (中国石油大学(华东)化学工程学院 重质油国家重点实验室 青岛 266580)
}

\begin{abstract}
摘要 环糊精是一种通过 $\alpha-1,4$-糖苷键将 $D$-吡喃葡萄糖单元连接在一起而形成的环状低聚糖，具有 “内疏水、外亲 水” 的刚性雉形空腔结构. 正是由于这个独特的 “内疏水、外亲水” 空间结构, 使得环糊精从被发现以来, 越来越受到 科学工作者的关注. 过渡金属催化剂作为重要的工业催化剂, 与环糊精体系结合可同时发挥金属的催化性能和环糊精 的分子识别和相转移等功能, 极大地改善其催化性能. 主要综述了环糊精参与的过渡金属催化的有机化学反应, 以金 属价态分类介绍了常见的 0 至 4 价过渡金属参与催化的有机反应，并对环糊精参与的金属共催化体系的未来发展前景 进行了展望, 预计今后该催化体系将会有更广阔的应用, 不断开发出更加高效和更有选择性的催化系统.
\end{abstract}

关键词 环糊精; 过渡金属; 催化; 反应; 合成; 配合物

\section{Transition Metal Catalyzed Organic Reaction Involving Cyclodextrin}

\author{
Chen, Yaqi \\ Gui, Xin \\ Duan, Zunbin \\ Zhu, Lijun \\ Xiang, Yuzhi \\ Xia, Daohong* \\ (State Key Laboratory of Heavy Oil Processing, College of Chemical Engineering, China University of Petroleum \\ (East China), Qingdao 266580)
}

\begin{abstract}
Cyclodextrin is a kind of cyclic oligosaccharide which is composed of the $D$-pyran glucose units connected with the $\alpha-1,4$-glycosidic bond. Cyclodextrin has the rigidly tapered cavity of hydrophobic inner and hydrophilic outer. Cyclodextrin has been attracted more and more attention from scientists since it was discovered, owning to its special space cavity of hydrophilic inner and hydrophilic outer. As an important industrial catalyst, transition metal catalyst can combine with the cyclodextrin system to simultaneously exert the catalytic properties of the metal and the molecular recognition and phase transfer of cyclodextrin, which greatly improves its catalytic performance. In this paper, the transition metal catalyzed organic reactions involving cyclodextrin are reviewed, and these reactions are described in terms of the metal valence from 0 to 4 . Finally, the development and foreground of these co-catalyst systems involving metal and cyclodextrin are prospected. It is expected that the catalytic system will have a wider application in the future, and a more efficient and selective catalytic system will be continuously developed.

Keywords cyclodextrin; transition metal; catalysis; reaction; synthesis; complexes
\end{abstract}

超分子化学(Supramolecular chemistry) ${ }^{[1]}$ 是一门新 型的前沿学科, 与诸多学科如配位化学、材料科学和生 命科学等相互交织. 超分子的形成主要是基于多个分子 相互识别的过程, 该过程不仅是主体分子与客体分子的 包合过程, 更重要的是主体分子对于底物的选择过程. 超分子包合是一种有目的、有选择的作用机制.

超分子化学研究的内容主要包括分子识别、自组装 和主客体化学等, 而主客体化学便是对超分子主体化合 物与其他小分子客体之间的作用进行探索, 例如冠醚对
金属阳离子的选择性包合、环糊精对芳香醛的包合催化 反应等 ${ }^{[2]}$. 环糊精(缩写为 $\mathrm{CD}$ ) 是在冠醚后超分子化学研 究最多的一类大环主体分子, 是一种通过 $\alpha-1,4-$ 糖苷键 将 $D$-吡喃型葡萄糖单元连接在一起而形成的环状低聚 糖, 主要包括 $\alpha$ - $\beta$-和 $\gamma$-环糊精(如图 1a $)^{[3]}$. 通过 X 射线 分析结果可以观察到环糊精具有一个特殊的刚性锥形 空腔结构(如图 1b). 环糊精外侧含有大量的羟基, 使得 环糊精具有较好的亲水性，可以溶解在水里，而内部不 具有羟基, 所以环糊精具有独特的疏水空腔. 正是由于

* Corresponding author. E-mail: xiadh@upc.edu.cn

Received September 6, 2018; revised December 3, 2018; published online January 18, 2019.

Project supported by the the National Natural Science Foundation of China (No. 21376265).

国家自然科学基金(No. 21376265)资助项目. 
这个独特的 “内疏水、外亲水” 空间结构, 使得环糊精 从被发现以来, 越来越受到科学工作者的关注.

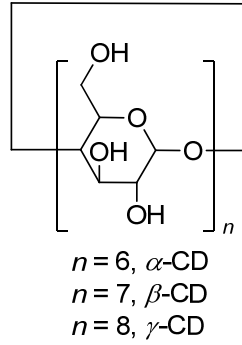

(a)

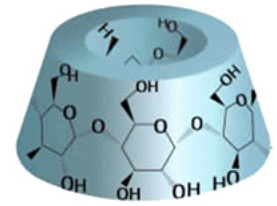

(b)
图 1 环糊精的分子结构(a)和三维结构示意图(b)

Figure 1 Molecular structure (a) and three-dimentional structure diagram (b) of cyclodextrin

在立体空间允许的情况下, 环糊精的空腔能够包含 各种疏水性的客体分子, 于是便形成一种独特的主客体 包合物，借此便能更好地将其应用于催化有机反应; 另 外, 环糊精因其无毒无害、价格低廉, 又具有良好的客 体识别性、优良的生物相容性与适当的空腔形状等优点, 且存在大量氢键作为活化基团, 使得环糊精催化有机反 应可以工业化、产业化.

然而天然环糊精的催化活性是有限的, 且在有机溶 剂中溶解性也较差, 极大地限制了其应用范围, 通常会 经过对其表面上的羟基进行修饰来完善其性能. 金属催 化剂作为重要的工业催化剂, 与环糊精体系结合可同时 发挥金属的催化性能和环糊精的分子识别和相转移等 功能, 极大地改善其催化性能. 近年来, 发表了基于不 同反应类型的环糊精催化反应方面的综述, 例如环糊精 衍生物在氧化、水解、还原、偶联等催化反应中的应用 进展综述 ${ }^{[4]}$. 而本文则主要综述了环糊精参与的过渡金 属催化有机反应, 并以金属价态分类对 $0 \sim 4$ 价常见过 渡金属参与催化的有机反应进行介绍.

\section{1 环糊精参与的纳米金催化有机反应}

纳米金(AuNPs) 由于具有独特的电学和光学特性, 在纳米科学和纳米技术领域是一项非常热门的研究, 并 具有广泛的应用潜力. 纳米金和大环超分子的复合更是 提供了一种新型的混合纳米材料, 并且有望带来新的特 性、新的功能和新的应用. Zhao 等 ${ }^{[5]}$ 合成了一种由环糊 精修饰的纳米金粒子(CD@AuNPs), 可用于传感、自组 装和级联催化. 单分散的纳米金粒径为 $15 \sim 20 \mathrm{~nm}$, 环 糊精作为还原剂和稳定剂, 以一种生态友好的一步胶体 合成法合成了混合的纳米材料. 首先, 纳米金粒子可以 作为基于客体置换反应产生的荧光传感的支架和能量 接收器. 其次, 大环超分子功能化的纳米金粒子可以有
效地进行组装，并利用四(4-羧基苯基)卟啉作为介体, 形成良好的一维、二维结构. 最后, 除了常规的主客体 交互作用之外, $\mathrm{CD} @ A u N P s$ 还展现出了令人意想不到 的催化活性一一同时具有葡萄糖氧化酶和辣根过氧化 物酶(HRP)这两种常见酶的模拟特性，在级联反应(首先 催化氧化葡萄糖生成葡萄糖酸和 $\mathrm{H}_{2} \mathrm{O}_{2}$, 然后 $\mathrm{H}_{2} \mathrm{O}_{2}$ 和四 甲基联苯胺被分别催化转化为 $\mathrm{H}_{2} \mathrm{O}$ 和相应的氧化物)中 被用作唯一的催化剂. 结合实验和密度泛函理论(DFT) 计算, Zhao 等推测 $\mathrm{CD} @$ AuNPs 的独特催化活性可能是 由环糊精分子的特殊拓扑结构以及由此产生的电子转 移效应引起. 该纳米材料的出现启示人们纳米金属材料 仍有许多 “隐藏” 的特性等待去发现，同时设计配体结 构也可能是一种新型的策略用来操纵纳米金属材料的 物理化学性质, 例如颗粒大小、晶面和催化特性.

Menuel 等 ${ }^{[6]}$ 则研究了环糊精和其它糖类添加剂在 机械合成纳米金粒子中的作用，以及它们催化硝基苯转 化为相应的苯胺产物中的作用. 在这里, 环糊精不仅可 以使纳米金粒子更加稳定，还可以通过超分子作用在固 体混合物中扩散基质，使化学反应定向到选择性生成苯 胺衍生物. Menuel 等仔细研究了影响纳米金的形成和苯 胺衍生物合成的因素, 研究表明, 糖类添加剂的性质和 苯取代基的位置均起到了很大的决定性作用，同时水也 在硝基的还原过程和超分子与底物的相互作用中起着 至关重要的作用. 此外, 该催化系统可以在连续三次使 用后回收利用，且催化活性不会造成明显的损失，这便 突出了机械化学、超分子化学和催化反应三者结合的特 别之处.

纳米金的化学和物理性质主要是取决于它们的大 小和形状, 而这些性质是可以通过改变形态和结构来调 节的. 在纳米金的不同形态中，树枝状的纳米金由于其 在多个领域中的潜在应用受到了极大的关注 ${ }^{[7,8]}$. Shanmugam 等 ${ }^{[9]}$ 在环糊精的辅助下将纳米金负载在氧 化石墨烯上，并利用电子显微镜观察了其生长机制，研 究表明环糊精修饰的氧化石墨烯(RGO)可通过电沉积技 术由最初的 $\mathrm{Au}^{3+}$ 最终形成树枝状纳米金(Scheme 1), 且 $\mathrm{CD}$ 修饰的 $\mathrm{RGO}$ 对于纳米金的成核和成长都起着至关 重要的作用. Shanmugam 等紧接着分析了纳米金的结构 和电化学性质, 结果显示纳米金同时也对亚硝酸盐和葡 萄糖的电解氧化反应具有协同催化作用, 经分析其催化 活性正是来源于其典型的树枝状纳米金结构，由于其具 有很多的活性位点，且在 RGO-CD 的支持下可充当微 小的纳米电极, 因此具有很高的电催化活性. 该合成方 法简单、有效、低成本，同样也适用于制备其他种类的 纳米金属，由此可见，该方法未来将在催化、生物传感 和纳米器件方面均具有很好的应用前景. 

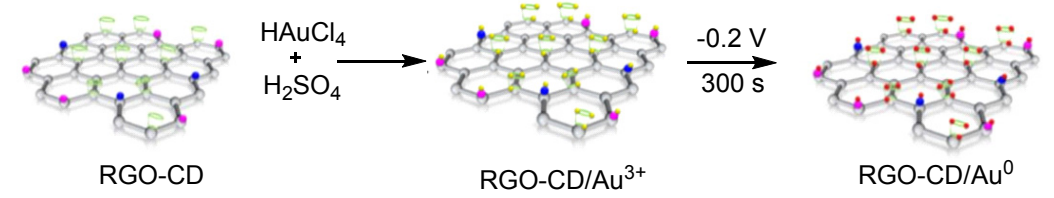

$\begin{array}{lc}-\mathrm{OH} & -\mathrm{Au}^{3+} \\ -\mathrm{COOH} & -\mathrm{Au}^{0}\end{array}-\mathrm{CD}$

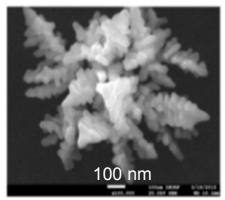

3D Au dendrite

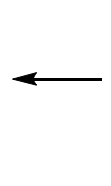

图式 1 树枝状纳米金的形成原理图

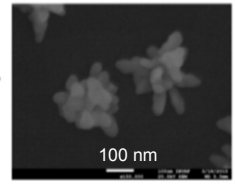

$\mathrm{CD}$ controlled growth
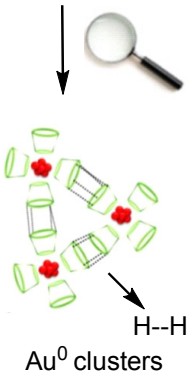

Protected by $C D$

Scheme 1 Schematic representation of the mechanism for the formation of 3D Au-NDs

\section{2 环糊精参与的一价 $\mathrm{Cu}$ 催化有机反应}

科学家们一直致力于寻找成本效益高和新的激活 技术以高效催化 $\mathrm{C}-\mathrm{C}$ 偶合反应和点击反应，尽管目前 的催化剂通过各种回收方式可以达到很高的周转率, 但 仍存在诸如由金属催化剂引起的产品污染和高成本等 缺点. Cravotto 等 ${ }^{[10]}$ 为了解决这一问题, 将 $\mathrm{Cu}^{1}$ 阳离子紧 密地嵌入到 $\beta-\mathrm{CD}$ 的腔体内, 并通过微波辐射进行物理 激活, 从而合成了一种新型的 $\beta-\mathrm{CD} / \mathrm{Cu}^{\mathrm{I}}$ 催化剂. 这种催 化剂具有独特的极性结构, 本身对电介质加热就十分敏 感的环糊精更是由于嵌入的阳离子而增强了这一效果, 因此它特别适用于微波辅助反应, 并且可以在短时间内 达到最高产量. Cravotto 等将此催化剂成功地应用于最 常见的点击反应中, 即苄基叠氮和苯乙炔的叠氮炔烃环 加成反应(Eq. 1), 反应温度为 $70{ }^{\circ} \mathrm{C}$ 时在 $100 \mathrm{~W}$ 的微波 辐射下 $10 \mathrm{~min}$ 便可使反应达到终点. 由于反应后金属的 损失可忽略不计, 因此该催化剂可循环使用, 且制备过 程简单, 符合绿色化学的要求.

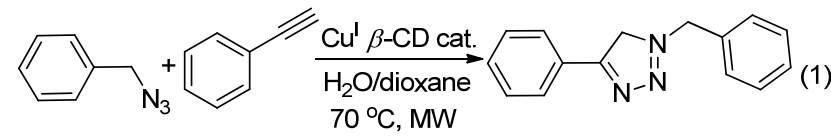

碘代三唑已知的合成方法是在亲电碘化试剂的存 在下由 $\mathrm{Cu}^{\mathrm{I}}$ 催化有机叠氮物和末端炔烃发生环加成作用 而生成 ${ }^{[11]}$, 而绿色化学的一项基本原则是提倡使用水作 为一种更安全、更环保的溶剂, 但是使用水作为反应介 质对有机合成化学家来说是一个巨大的挑战, 因为有机 化合物在水中的溶解度很低, 然而环糊精可以通过包合 有机材料提高其在水中的溶解性. 基于这些研究, Dheer 等 ${ }^{[2]}$ 研究了一种新型的 $\mathrm{CuI}($ 碘化亚铜) $/ \beta-\mathrm{CD}$ 催化剂, 并 首次用于水中区域选择性合成 5 -碘-1,2,3-苯并三唑、2-
碘咪唑吡嗪和 2-碘苯咪唑噻唑(Scheme 2), 该方法的优 点是条件温和、操作简单、产量高、底物通用性高以及 选择水作为绿色溶剂.

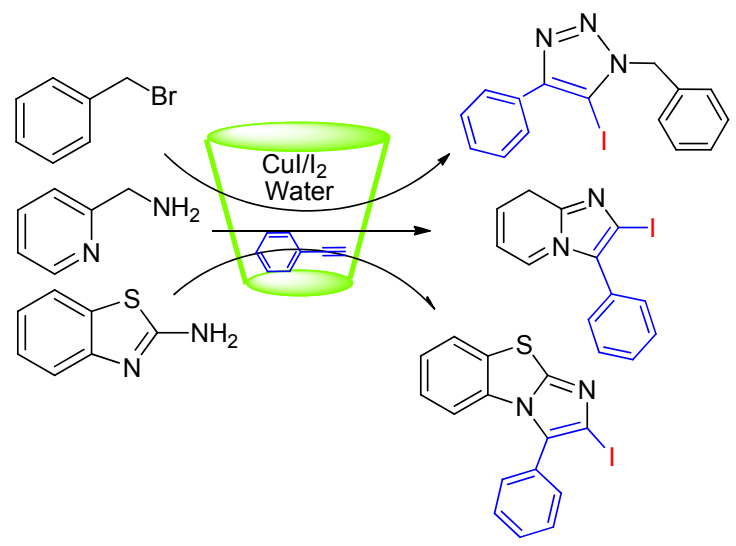

图式 $2 \mathrm{CuI}($ 碘化亚铜 $) / \beta-\mathrm{CD}$ 催化合成 5-碘-1,2,3-苯并三唑、 2-碘咪唑吡嗪和 2-碘苯咪唑噻唑

Scheme $2 \mathrm{CuI} / \beta$-CD-catalyzed regioselective synthesis of 5-iodo-1,2,3-triazoles, 2-iodoimidazopyridines, and 2-iodobenzoimidazothiazoles

Patil 等 ${ }^{[13]}$ 开发了一种常温下在水溶液中铜环糊精 催化醇的氧化工艺, 首次报道了在氧化过程中形成了 $\mathrm{Cu}_{2} \mathrm{O}-\mathrm{CD}$ 纳米结构. 研究证明了不同铜盐、 $\mathrm{CD}$ 和碱基 对纳米颗粒的形态具有影响, 该催化剂对一锅化三组分 的丙炔氨化反应显示出了良好的催化效率, 收率可高达 $85 \%$ ，且反应后通过添加乙酸乙酯回收粗产物即可实现 催化剂的再利用. 他们还对使用后的催化剂进行了 $\mathrm{XRD}$ 分析, 以探测其成分和结构的变化, XRD 图谱分析 表明，峰值没有变化即没有新的晶相物种产生，从而证 明催化剂没有发生任何变化, 这些研究解释了该催化过 程可能是一种表面介导催化作用. 


\section{3 环糊精参与的二价金属催化有机反应}

\section{1 环糊精参与的 $\mathrm{Cu}$ " 催化有机反应}

在分析金属与环糊精相互作用的文献资料时，可以 看到大部分都是涉及到二价金属, $\mathrm{Cu}^{\mathrm{II}}$ 更是其中的 “佼 佼者”。早在 1927 年, Messmer ${ }^{[14]}$ 便首次在碱性溶液中 将 $\mathrm{Cu}^{\mathrm{II}}$ 配位在 $\beta$-环糊精上.

在过去的 20 年中, “点击化学” 在学术界以及工业 上都引起了相当多的关注, 因为它能从简单的基质中高 效地制备更复杂的产品 ${ }^{[15 ~ 17]}$. 其中, 铜催化的叠氮炔烃 环加成反应 $(\mathrm{CuAAC})$ 便是最原始也是最著名的一个例 子 ${ }^{[18,19]}$. CuAAC 法的特点是区域选择性高和生物相容性 良好，能在缓和的条件下高效地合成 1,2,3-苯并三坐衍 生物, $\mathrm{Cu}^{\mathrm{II}}$ 在该反应中起到了至关重要的作用. 同样 CuAAC 法也可用于其他的有机反应 ${ }^{[20 ~ 24]}$, 经 Sharpless 和 Medal 研究发现, 不同的铜离子化合物, 例如 $\mathrm{CuI}^{[25]}$ 、 $\mathrm{Cu}(\mathrm{OAc})_{2}{ }^{[26]} 、 \mathrm{CuOTf} \cdot \mathrm{C}_{6} \mathrm{H}_{6}{ }^{[27,28]}$ 和 $\left[\mathrm{Cu}(\mathrm{phen})\left(\mathrm{PPh}_{3}\right)_{2}\right]-$ $\mathrm{NO}_{3}{ }^{[29]}$, 都曾用于催化此类反应. 然而, 尽管这些均相 催化剂具有如此优良的特性, 它们的应用仍然受到限 制, 因为它们具有不易分离回收、含有细胞毒性和在反 应过程中易被破坏等缺点. 为了克服这些问题, 研究者 们将更多的关注放在了非均相催化剂上, 因为它们相比 于同类型的均相催化剂更容易分离回收, 且在自然界中 是低毒的 ${ }^{[30 ~ 32]}$. 通过将铜离子固载于不溶性的载体之 上, 便可以取两者之所长. 如今, 人们越来越热衷于将 对环境无害的物质作为非均相催化剂 ${ }^{[33 \sim 35]}$. Nie 等 ${ }^{[36]}$ 首 次将 $\mathrm{Cu}^{\mathrm{II}}$ 修饰的 $\gamma-\mathrm{CD}$ 负载在氮化硼上(h-BN@ $\gamma-\mathrm{CD} @-$ $\left.\mathrm{Cu}(\mathrm{OAc})_{2}\right)$ (图 2), 这种可回收再重复利用的非均相催化 剂可用于合成 1,2,3-苯并三唑衍生物. 且该催化剂具有 良好的官能团耐受性, 可以高收率催化水相中的反应. 此外, 催化剂还可以直接通过离心回收, 循环使用 7 次 以后收率依旧很高.

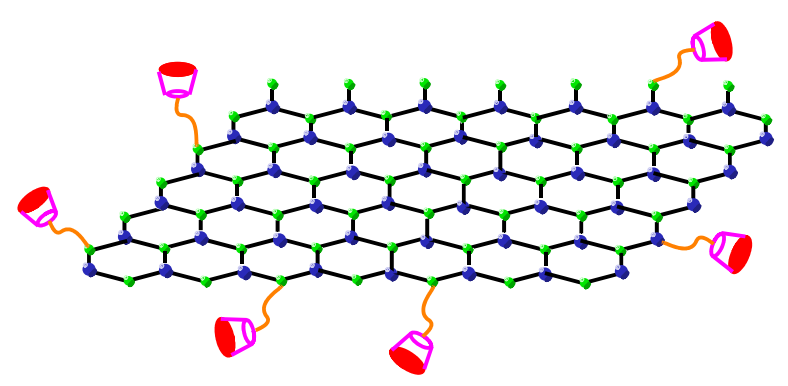

$\because$ B : N Y : APTMS-Amber Acid

: $\mathrm{y}-\mathrm{CD} @ \mathrm{Cu}(\mathrm{OAC})_{2}$

图 2 h-BN@y-CD@ $\mathrm{Cu}(\mathrm{OAc})_{2}$ 催化剂结构

Figure 2 Structure of h-BN@ $\gamma-\mathrm{CD} @ \mathrm{Cu}(\mathrm{OAc})_{2}$ catalyst

环糊精是一种很好的能够包合客体分子的主体化 合物, 大范围地应用于水相反应中. 由于其能够识别不
同的小分子和改善有机小分子在水中的溶解性，环糊精 经常被用作水相催化剂. 近些年以来, 嗍氢化反应引起 了学术界极大的关注. 在大量文献报道中, 铜催化的嗍 氢化反应更是尤为重要. 一方面, 研究发现使用过渡金 属催化剂可以显著加快硼氢化反应的速率，甚至能够实 现立体选择性或反应区域的反转; 另一方面，与贵金属 如 Pd、Pt、Rh、Ir 等相比，铜具有价格低、毒性小等优 点. Yao 等 ${ }^{[37]}$ 报道了一种在水介质中条件温和、环境友 好型的铜催化的末端炔烃嗍氢化反应, 在环糊精一双吡 啶配体(CD-1) (Eq. 2)的存在下, 可进行区域选择性控 制. 该反应可成功应用于保护失活的末端炔烃，且该配 体至少可以回收重复利用 5 次后仍具有催化活性. 这是 首次报道的环糊精衍生物能够催化炔烃进行区域选择 性硼氢化反应，成为从简单的末端炔烃中获得乙烯基硼 烷的有效途径.

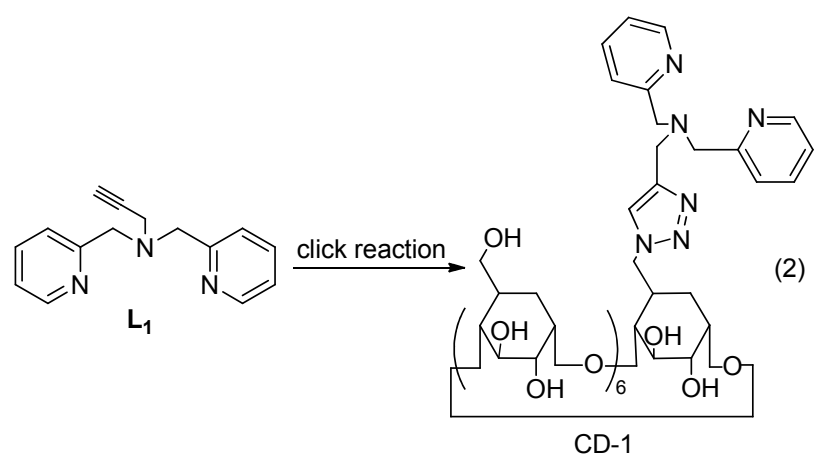

Zhang 等 ${ }^{[38]}$ 研究合成了一种新型环糊精衍生物, 是 由 $\mathrm{N}$-杂环化合物包裹而形成的环糊精复合物(ICyD), 分别由 $\alpha-\mathrm{CD} 、 \beta-\mathrm{CD}$ 得到了 $\alpha-\mathrm{ICyD} 、 \beta-\mathrm{ICyD}$, 在铜催化 的硼氢化反应中发生了相反的区域选择性. 反应的结果 主要取决于环糊精的性质, $\alpha-\mathrm{CD}$ 衍生物提供的是末端 炔烃的线性产物, 而 $\beta$ - $\mathrm{CD}$ 衍生物提供的则是支链产物 (Scheme 3). 这两种催化剂的区别在于其机制有所差异: 传统的并行机制和新型的正交机制，腔体形状的差异性 不仅能引起一个区域选择性开关，同时还能产生一个机 械开关. ICyD 中的金属表面是由环糊精支架包裹着的, 腔体内附加金属的位置迫使外部的配体受其形状影响 而与金属中心发生相互作用, 因此, 这种特殊的结构导 致在催化环异构化反应时诱发了受形状依赖的立体选 择性和区域选择性. $\alpha$-ICyD 和 $\beta$-ICyD 的结构分析表明, 这两种环糊精衍生物在形状上有着明显的差异, 尤其是 在金属存在的情况下.

Kaboudin 等 ${ }^{[39]}$ 将环糊精和金属铜有效结合，合成 了一种高效的纳米催化剂 $\mathrm{Cu}^{\mathrm{II}}-\beta-\mathrm{CD}$, 可用于在均相条 件下通过偶联反应一锅法制得 1,2,3-苯并三唑化合物. $\mathrm{Cu}^{\mathrm{II}}-\beta-\mathrm{CD}$ 是一种具有良好稳定性的双核复合材料，可 


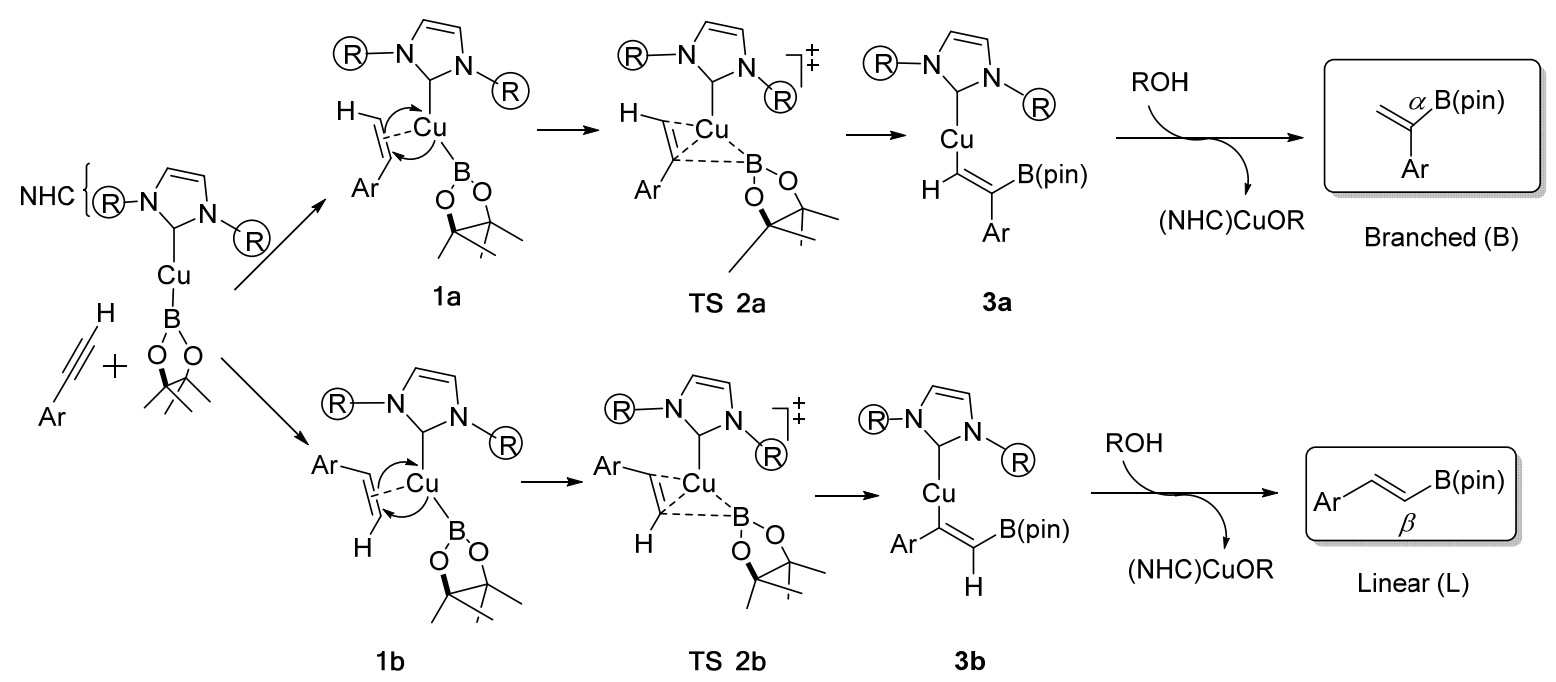

图式 3 线性产物和支链产物的形成途径

Scheme 3 The formation of the branched or linear adducts

以通过将硫酸铜溶液加入到 $\beta$-环糊精和 $\mathrm{NaOH}$ 的溶液 中获得. 尽管该种催化剂具有很多优点, 例如良好的活 性和选择性以及较高的转化率, 广泛应用于各行各业, 但是将这种可溶性的催化剂与产品和反应物分离开来 仍然很困难. 因此, 在此基础上, Kaboudin 等 ${ }^{\left[{ }^{400}\right]}$ 制备了 一种高效的、易于回收、可重复利用的 $\mathrm{Fe}_{3} \mathrm{O}_{4}$ 磁性纳米 粒子支撑的 $\mathrm{Cu}^{\mathrm{II}}-\beta-\mathrm{CD}$ 复合物催化体系 $\left(\mathrm{Fe}_{3} \mathrm{O}_{4}-\beta\right.$ $\left.\mathrm{CD}-\mathrm{Cu}_{2}\right)$, 可通过催化芳基硼酸发生自身偶联反应，从 而高效地合成联苯化合物, 还可以直接在室温下以水为 介质催化芳基硼酸、叠氮化钠与炔烃通过环化反应合成 1,2,3-苯并三唑. 经检验, 催化剂通过磁铁回收循环使 用 4 次后催化活性仅略有损失.

\section{2 环糊精参与的 $\mathrm{Pd}$ " 催化有机反应}

$\mathrm{Pd}^{\mathrm{II}}$ 可催化交叉耦合反应形成 $\mathrm{C}-\mathrm{C}$ 键, 此反应广 泛应用于合成和医疗化学中以制备具有巨大治疗潜力 的新型药物 ${ }^{[1,42]}$. 一般来说, 磷配体会用来激活钯元素, 但是, 它们对空气和湿度都很敏感, 限制了催化剂的重 复使用, 而且在水介质中反应也会生成许多副产物, 极 大地限制了其应用 ${ }^{[43,44]}$. 因此, 钯催化系统中避免使用 磷化氢被认为是有机合成领域中最具有挑战性的研究 之一. Raihana 等 ${ }^{[45]}$ 将 $\mathrm{Pd}(\mathrm{II})$ 离子负载在水溶性的吡啶修 饰的 $\beta$-CD 上( $\left.\mathrm{Pd}^{\mathrm{II}} @ \mathrm{Pyr}: \beta-\mathrm{CD}\right)$, 研究表明此复合物可高 效催化水介质中的 Suzuki-Miyaura、Heck $\mathrm{C}-\mathrm{C}$ 耦合反 应, 利用少量的 $\mathrm{Pd}^{\mathrm{II}} @ \mathrm{Pyr}: \beta-\mathrm{CD}$ 便可以高收率催化各种 卤代芳烃参与的耦合反应, 包括氯化物和苯硼酸、氯化 物和苯乙烯发生的反应. 这种均相催化剂可在保证其催 化活性的基础上至少再循环使用 6 次以上.

Qi 等 ${ }^{[46]}$ 设计了一种利用非共价键结合来设计和制
备水溶性金属催化剂的新方法，是将疏水性的钯二吡唑 复合物负载于金刚烷上和相互补的亲水性反应形成了 配合物(Ad-L- $\left.\mathrm{PdCl}_{2} @ \beta-\mathrm{CD}\right)$. 这一催化剂对于亲水性芳 基硼酸和芳基澳代物在有机溶剂中发生的 Suzuki-Miyaura 耦合反应具有超高的催化活性(Scheme 4). 相比较之下, 在相同的反应条件下, 单纯的钯复合 物 $\left(\mathrm{Ad}-\mathrm{L}-\mathrm{PdCl}_{2}\right)$ 的催化效率大大降低. 反应完毕后, 可 以 $n-\mathrm{Bu}_{4} \mathrm{NBr}$ 为稳定剂对其进行离心分离回收再利用, 但该催化剂负载量较高. 为降低贵金属的使用量, Zhou 等 ${ }^{[47]}$ 最近又新合成了一种低钯负载量(仅 $0.00084 \mathrm{wt} \%$ ) 的催化剂- $-\mathrm{PdCl}_{2}(\mathrm{Ln} @ \beta-\mathrm{CD})$, 显示出了更良好的催 化性能, 可在水中高效催化芳基嗍酸和芳基卤化物的 Suzuki 耦合反应. 实验表明, 当 $\mathrm{K}_{3} \mathrm{PO}_{4} \cdot 7 \mathrm{H}_{2} \mathrm{O}$ 存在时, 以 $\mathrm{PdCl}_{2}(\mathrm{Ln} @ \beta-\mathrm{CD})$ 为催化剂催化芳基溴化物, $4 \mathrm{~h}$ 后分离 得到的联芳烃衍生物的收率便可以达到 $80 \% \sim 100 \%$, 而控制实验表明没有 $\mathrm{PdCl}_{2}(\mathrm{Ln} @ \beta-\mathrm{CD})$ 存在的条件下只 能获得微量的产品. 为了进一步研究该催化剂中三种配 体的影响, 又进行了一系列比较实验, 发现只有微量 $\beta-\mathrm{CD} 、 \mathrm{PdCl}_{2}$ 、邻溴甲苯和苯基硼酸作为反应底物时, 只 能获得 $6 \%$ 的收率, 该结果更清楚地表明了该复合型催 化剂的重要性.

Guo 等 ${ }^{[48]}$ 也合成了一种金属钯修饰形成的环糊精 复合物(DACH-Pd- $\beta-\mathrm{CD})$, 并对其进行了表征. 结果表 明, 此催化系统在常温下、水溶液中存在硼氢化钠的情 况下, 对不同的硝基苯类化合物还原成相应的苯胺具有 较高的催化性能, 所需产品的产量最高可达 99\% (Eq. 3). 此外, 催化剂可以很方便地进行分离回收, 并且循 环使用 5 次之后仍然可以维持较高的催化活性. 

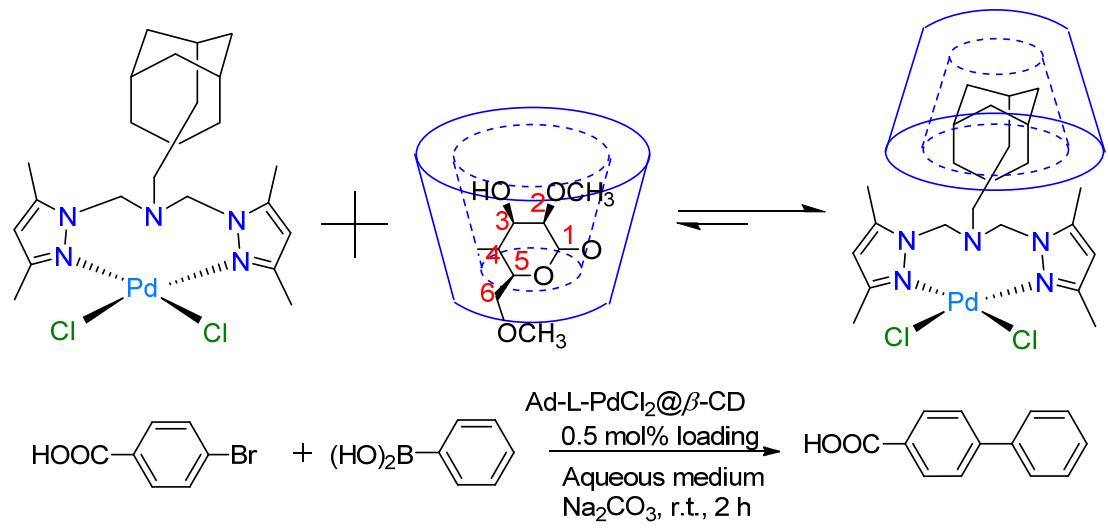

图式 4 Ad-L-PdCl $@ \beta$-CD 催化的 Suzuki-Miyaura 耦合反应

Scheme 4 The Suzuki-Miyaura coupling reaction catalyzed by Ad-L-PdCl ${ }_{2} @ \beta$-CD

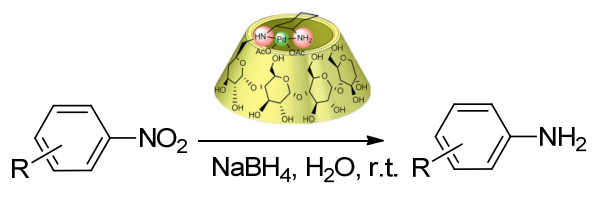

(3)

近年来, 许多传统催化剂被报道用于不同条件下的 转移氢化反应，这些报道大多使用高温、过量的不可回 收催化剂和有毒试剂. 在此背景下, Imran 等 ${ }^{[49]}$ 合成了 一种水溶性的 $\mathrm{Pd}^{\mathrm{II}} @ \mathrm{PyPDA}: \beta-\mathrm{CD}$ 复合物, 可作为绿色 环保的均相催化剂, 在温和的反应条件下以异丙醇为还 原剂, 将芳香族酮加氢转化为仲醇. 该催化剂重复使用 5 次后, 其催化活性仅略有降低. 作者提出了一种可行 的氢化机理. 首先, 异丙氧基和 $\mathrm{Pd}^{\mathrm{II}} @$ PyPDA: $\beta$-CD 结 合, 紧接着发生 $\beta$ 位的氢消除生成了一个瞬态的氢化物, 然后芳香族酮插入到 $\beta$ - $\mathrm{CD}$ 腔体中生成了一个中间产物, 最后迅速与异丙醇发生转移氢化反应, 从而触发第二个 催化循环. 该催化剂还有许多显著的优点, 如制备简 单、稳定性好、底物选择性强、易回收、可重复利用, 因 此在其他反应和工业应用中也有极大的潜在用途.

\section{4 环糊精参与的三价 Fe 催化有机反应}

血红素不仅是最丰富、使用最广的金属卟啉催化剂 之一, 也是多种金属酶的重要辅因子. 近年来, 许多含 血红素的金属酶在生物合成和生物分析化学中都得到 了相当大的关注 ${ }^{[50]}$. 金属催化的有机胺和 $\alpha$-重氮酯的 $\mathrm{N}-\mathrm{H}$ 插入反应为含有 $\mathrm{C}-\mathrm{N}$ 键活性分子的合成提供了 简单易行的方法, 然而这种反应大多数使用有机溶剂, 并且需要严格的反应条件, 另一方面, 由于水溶液中的 $\mathrm{O}-\mathrm{H}$ 键和底物的水不溶性, 酶催化的卡宾体转换反应 仍然很难发生于水介质中 ${ }^{[51]}$. 同样的问题也阻碍了血 红素催化卡宾体嵌入有机胺中, 并且由于卟啉环的疏水 性, 血红素在水介质中的催化活性通常也会有所降低. 此种情况, “内疏水, 外亲水” 的环糊精便可以为血红
素提供有利的微环境以提高有机基质的溶解度. $\mathrm{Xu}$ 等 ${ }^{[52]}$ 利用环糊精协助血红素在水介质中成功催化了 $\alpha$ 重氮酯与芳香胺的 $\mathrm{N}-\mathrm{H}$ 键插入反应(Eq. 4), 产率可高 达 $96 \%$ ，且反应体系对不同的芳香胺均展现出了良好的 兼容性. 同时也在设计控制实验的基础上初步提出了反 应机理(Scheme 5), 首先, 重氮化合物经过亲电加成, 通过失去一个 $\mathrm{N}_{2}$ 分子来协调血红素的不饱和 $\mathrm{Fe}^{3+}$ 中 心, 形成了一个血红素卡宾配合物; 然后，通过胺基的 亲核进攻产生了 $\mathrm{N}-\mathrm{H}$ 键嵌入物. 这种以水为介质的催 化体系在卡宾体转化反应中占有很大的优势, 血红素与 环糊精的协同作用也为血红素酶应用于重氮酯的转化 反应提供了一种初步的可能性.<smiles>COC(=O)C(Nc1ccccc1)c1ccccc1</smiles>
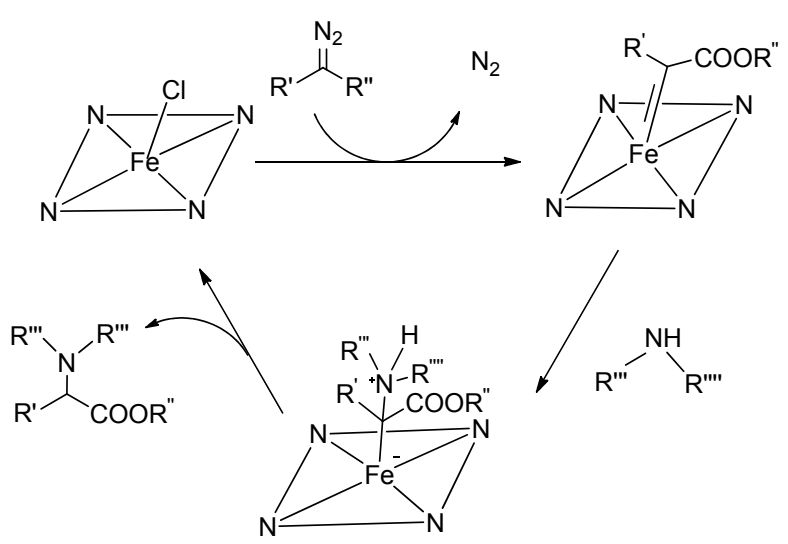

图式 5 环糊精协助血红素催化的 $\alpha$-重氮酯与芳香胺的 $\mathrm{N}-\mathrm{H}$ 键插入反应机理

Scheme 5 Mechanism of hemin-catalyzed, cyclodextrin-assisted $\mathrm{N}-\mathrm{H}$ insertion reaction of $\alpha$-diazo ester into aromatic amines 
Wang 等 ${ }^{[53]}$ 也以一锅法制备了 $\mathrm{Fe}_{3} \mathrm{O}_{4} @ \beta-\mathrm{CD}$ 磁性纳 米复合材料, 并利用其催化污染物的降解 (Scheme 6). 在这里, 4-氯苯酚和氯苯被选中作为模型污染物, 它们 通常被添加到生活中常见的除草剂、杀菌剂、杀虫剂和 防腐剂中. Wang 等对 $\mathrm{Fe}_{3} \mathrm{O}_{4} @ \beta-\mathrm{CD}$ 复合材料的理化性能 进行了表征, 并根据 $\mathrm{pH}$ 值、 $\mathrm{H}_{2} \mathrm{O}_{2}$ 浓度和催化剂用量等 关键变量的影响, 对其催化性能进行了评价, 同时也对 反应动力学、材料稳定性和降解机理进行了评估. 结果 表明, $\mathrm{Fe}_{3} \mathrm{O}_{4} @ \beta$ - $\mathrm{CD}$ 比 $\mathrm{Fe}_{3} \mathrm{O}_{4}$ 表现出更高的催化活性, 对 于 4-对氯苯酚的降解速率分别为 0.0373 和 0.0162 $\mathrm{min}^{-1}$, 这可能是由于形成了三元复合物, 其允许所产 生的羟基自由基直接攻击 4-对氯苯酚, 同时也增加了有 机污染物的溶解度. $\mathrm{Fe}_{3} \mathrm{O}_{4} @ \beta$ - $\mathrm{CD}$ 对于催化氯苯的降解 也表现出了较高的催化活性, 其降解速率为 0.0392 $\mathrm{min}^{-1}\left(\mathrm{Fe}_{3} \mathrm{O}_{4}\right.$ 催化的降解速率为 $\left.0.0099 \mathrm{~min}^{-1}\right)$, 可能由 于 $\mathrm{Fe}_{3} \mathrm{O}_{4} @ \beta-\mathrm{CD}$ 复合材料所具有的协同效应. 此外, $\mathrm{Fe}_{3} \mathrm{O}_{4} @ \beta-\mathrm{CD}$ 还表现出稳定的机械强度和较好的重复利 用性. 仅含有 $\mathrm{Fe}^{3+}$ 或无定形氧化铁的矿物质在催化剂表 面的稳定性较差、溶解度较高, 因此, 稳定性是多相催 化剂的一个重要特性. 为此, Wang 等对催化剂的稳定性 做了测试, 为了验证溶液中溶解的铁离子对反应的影 响, 在相同反应条件下, 以最大浸出铁离子量为基础进 行了分批实验. 最后的研究表明, $\mathrm{H}_{2} \mathrm{O}_{2}$ 的活化即 $\cdot \mathrm{OH}$ 的 生成, 主要还是归因于 $\mathrm{Fe}_{3} \mathrm{O}_{4} @ \beta-\mathrm{CD}$ 的催化作用, $\cdot \mathrm{OH}$ 的生成主要发生在固体催化剂的表面, 这样, 吸附在催 化剂表面的有机物就可以直接被・OH 攻击降解, 便不再 需要经过浸出的 $\mathrm{Fe}$ 离子催化降解. 因此, 该复合材料可 以有效地回收, 不会在溶液中损失大量的铁离子, 且催 化剂的结构和组成也不会发生明显变化. 同时, 通过对 4-对氯苯酚降解的中间产物和氯离子含量的分析, 提出 了污染物可能的降解机理, 并根据密度泛函理论(DFT) 探究了污染物和环糊精之间的主一客体相互作用, 阐述 了由于环糊精的特定空间选择性而导致了降解中间产 物的单一性.

\section{5 环糊精参与的四价 $\mathrm{Ti}$ 催化有机反应}

$\mathrm{TiO}_{2}$ 是一种储量丰富、价格低廉、环境友好的材料, 结构稳定、有较大的能带隙 $(3.2 \mathrm{eV})$, 然而其光响应范围 过于狭窄, 此时由 $\mathrm{TiO}_{2}$ 和石墨烯制成的纳米复合材料 便可以解决这一问题. 石墨烯在没有功能化的情况下维 持其稳定的分散状态是十分困难的, 这是由于其自身会 发生聚集和重叠, 而在 $\pi-\pi$ 相互作用和范德华力作用下 其表面积将会减小，这便限制了氧化石墨烯的实际应 用. 因此, 石墨烯的表面功能化更显得尤为重要. 据报 道, 在极性溶剂中, 石墨烯和环糊精的超分子体系具

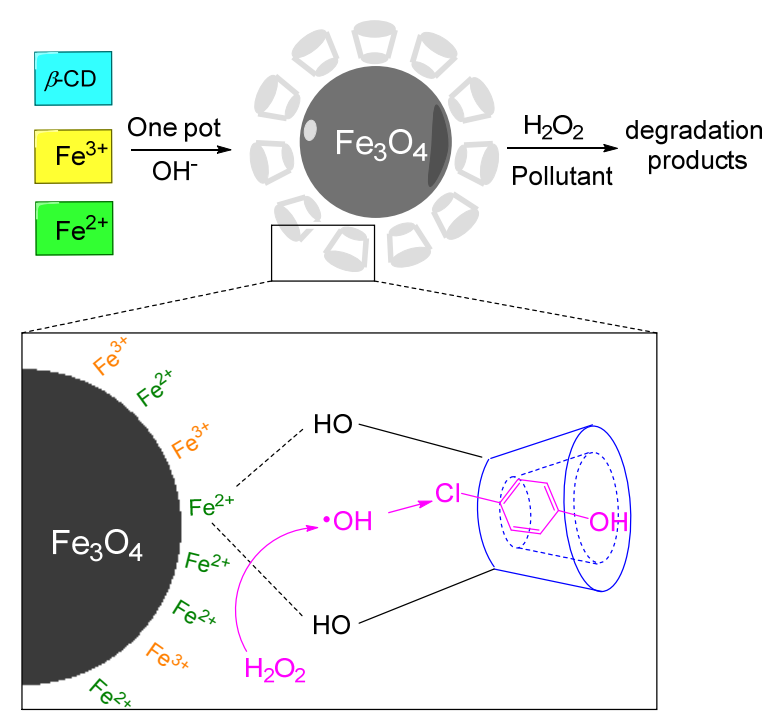

图式 $6 \mathrm{Fe}_{3} \mathrm{O}_{4} @ \beta-\mathrm{CD}$ 的制备过程和降解过程原理图 Scheme 6 Preparation process of $\mathrm{Fe}_{3} \mathrm{O}_{4} @ \beta$-CD and schematic diagram of degradation process

有很高的分散性 ${ }^{[54]}$. 该超分子体系可稳定分散于水溶 液中，可为金属盐提供原位还原以形成超分子金属纳米 复合材料. Sharavath 等 ${ }^{[55]}$ 首次在 $90{ }^{\circ} \mathrm{C}$ 低温下用一种简 单的水相法合成了 $\mathrm{TiO}_{2}-\beta$ - $\mathrm{CD}$-石墨烯 $\left(\mathrm{TiO}_{2}-\mathrm{CD} @ \mathrm{GNS}\right)$ 纳米复合材料(Scheme 7), 利用 $\beta$-CD 作为稳定剂将石墨 烯固定于水相中, 防止其发生聚集, 从而促进了 $\mathrm{TiO}_{2}$ 纳米粒子在石墨烯纳米薄片上的原位自组装过程. 这种 新型材料比原来的 $\mathrm{TiO}_{2}$-氧化石墨烯材料具有更好的能 量储存能力和更高的光催化活性, 在可见光下使用 $\mathrm{TiO}_{2}-\mathrm{CD} @ \mathrm{GNS}$, 可在 $25 \mathrm{~min}$ 内实现亚甲基蓝的 100\%光 降解, 由此可见其高效的光催化活性. 研究表明这可能 是由于增加了可见光的吸收度和通过 $\mathrm{Ti}-\mathrm{O}-\mathrm{C}$ 键在 $\mathrm{Ti}$ 和 C 中进行电子转移, 从而极大地阻碍了由光产生的电 子空穴对的重组.

Mohamed 等 ${ }^{[56]}$ 也合成了一种 $\mathrm{TiO}_{2}-\mathrm{CD}-\mathrm{GN}$ 纳米复 合材料, $\mathrm{TiO}_{2}$ 纳米颗粒通过光照和环糊精的协助固定在 石墨烯纳米薄片上. 研究表明, 该复合材料也可用于可 见光下 4 -氯苯酚的光降解, 且催化活性远高于 $\mathrm{TiO}_{2}$. Subramanian 等 ${ }^{[57]}$ 则制备了一种 $\mathrm{TiO}_{2}-\mathrm{CD}$ 复合材料用于 光降解 4-硝基苯酚, 环糊精同样也大大增强了 $\mathrm{TiO}_{2}$ 的 光催化活性, 通过 UV-DRS、FE-SEM 和 PXRD 分析, 主 要可归结为两个关键因素: (1) $\mathrm{TiO}_{2}-\mathrm{CD}$ 的带隙能量比相 对应的 $\mathrm{TiO}_{2}$ 要低; (2) $\mathrm{TiO}_{2}-\mathrm{CD}$ 中 $\mathrm{TiO}_{2}$ 的相结构和表面 形态都保持不变. 此外, $\beta$-CD 还可以作为 4-硝基苯酚和 $\mathrm{TiO}_{2}$ 之间的一个桥梁, 从而促进了它们之间的相互作 用.

Sun 等 ${ }^{[58]}$ 采用光诱导装配法合成了新型光催化复合 材料 $\mathrm{WF} / \beta-\mathrm{CD} / \mathrm{TiO}_{2}$ (WF 为一种天然纤维素的合成物), 


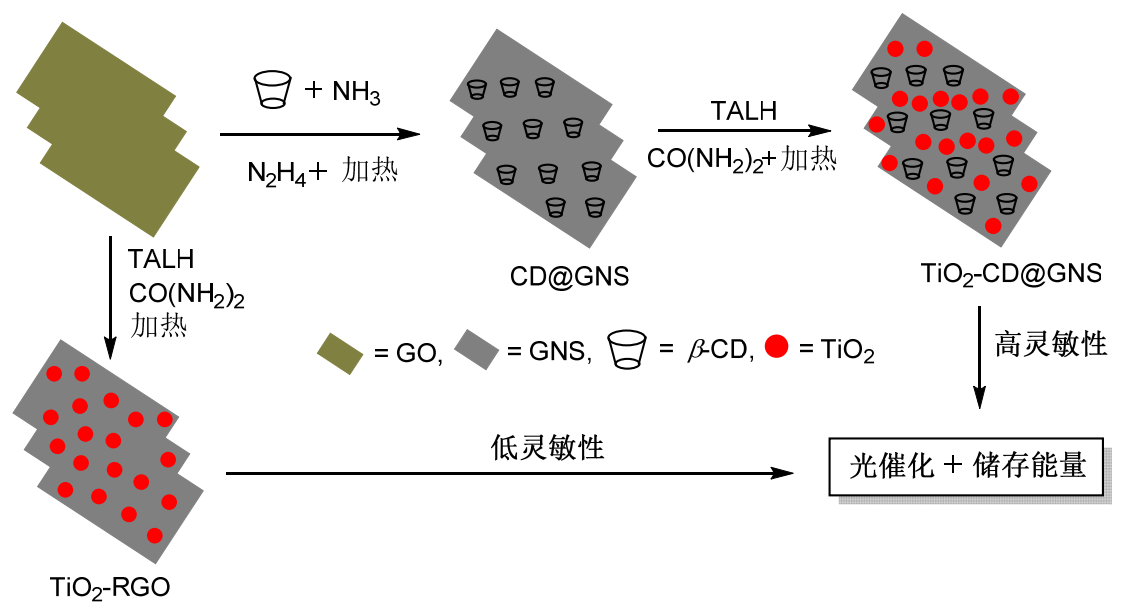

图式 $7 \mathrm{TiO}_{2}-\mathrm{CD} @ \mathrm{GNS}$ 纳米复合材料的制备过程

Scheme 7 Preparation process of $\mathrm{TiO}_{2}-\mathrm{CD} @$ GNS nanocomposite

并评估了其对甲基橙 $(\mathrm{MO})$ 的光催化降解效率. 在该材 料中, $\beta$-CD 用来捕获光催化底物, 并将其转移到 $\mathrm{TiO}_{2}$ 表 面, 以此来提高降解效率(Scheme 8). 他们利用酚酞探 针技术和反向滴定法, 测得 $\mathrm{WF} / \beta-\mathrm{CD} / \mathrm{TiO}_{2}$ 中分别含有 $3.5 \%$ 的 $\beta$-CD 和 $0.0581 \mathrm{~g} / 0.1 \mathrm{~g}$ 的 $\mathrm{TiO}_{2}$, 它可以在 $21 \mathrm{~min}$ 内完全降解 $100 \mathrm{~mL}$ 浓度为 $0.1 \mathrm{mmol} \cdot \mathrm{L}^{-1}$ 的甲基橙溶 液. WF 的加入虽然对 $\beta-\mathrm{CD} / \mathrm{TiO}_{2}$ 的光催化活性影响不 大, 但为进一步将 $\mathrm{TiO}_{2}$ 制备为高效的光催化剂提供了 理想的生物质载体. 另外, 在进行了 5 次循环试验后, 降解效率仍高达 $86 \%$, 明显高于同样条件下的 $\beta-\mathrm{CD} /$ $\mathrm{TiO}_{2}$, 表现出良好的再生性能. 上述所提到的几种环糊 精 $-\mathrm{TiO}_{2}$ 复合型催化剂均经过热重分析, 结果表明均具 有较好的热稳定性.

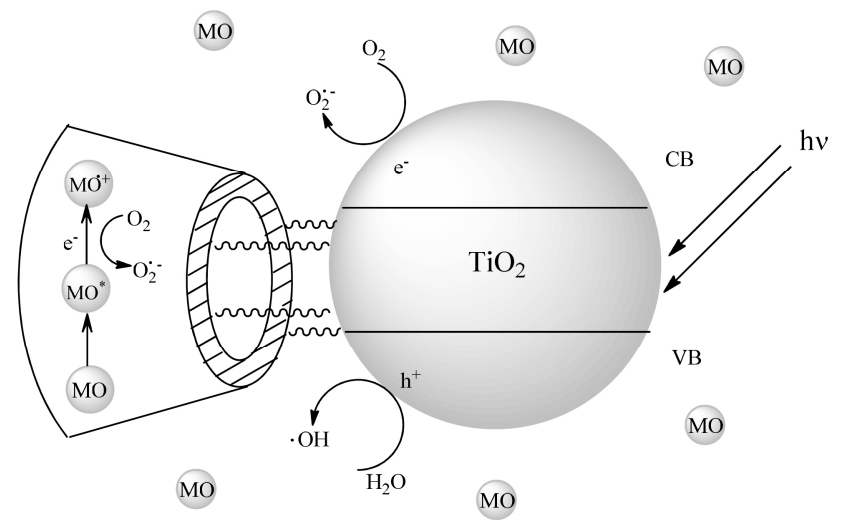

图式 $8 \beta-\mathrm{CD}$ 在 $\mathrm{TiO}_{2}$ 光催化反应中的作用解释图 Scheme 8 Interpretation map of the rule of $\beta$-CD in photocatalytic reactions of $\mathrm{TiO}_{2}$

\section{6 总结与展望}

本文系统介绍了环糊精参与的过渡金属催化的有 机化学反应, 重点探讨了环糊精参与的常见 0 至 4 价过
渡金属催化的有机反应. 由于环糊精腔体外表面上存在 许多可作为活化基团的羟基，因此可以对其进行修饰， 从而获取到具有更加优良性能的环糊精衍生物. 环糊精 及其衍生物与金属催化剂的结合能够更加高效地催化 有机反应在水相中完成, 有效地避免了使用成本高或具 有污染性的有机溶剂, 同时, 环糊精本身无毒无害、价 格低廉, 绿色环保, 更契合当今 “绿色化学” 的理念. 环 糊精参与的过渡金属催化体系中环糊精最重要的作用 便是将底物包结于其 “内疏水、外亲水” 的空腔中, 这 便可以做到在为底物提供一个疏水环境的同时, 让此包 合物又溶于水中, 借此该包合物便可以顺利地在水相中 实现有机合成转化. 综上所述, 环糊精参与的过渡金属 催化体系优点如下: (1)催化体系的反应速率和区域选择 性有了显著提升; (2)可设计制备出新型的水溶性催化 剂, 绿色环保; (3)环糊精与底物形成包合物, 有利于加 强金属催化剂和底物之间的接触, 使底物更加易于反 应. 但由于环糊精本身是低聚糖类物质，高温下易分解， 因此, 如何对环糊精进行合理的修饰才能使其更加稳定 也是本领域面临的一大难题. 今后该领域的关键是进一 步研究环糊精参与的过渡金属催化有机反应的机理, 以 便改进或调控催化性能, 加快环糊精参与的过渡金属催 化体系的大规模工业化应用. 因此希望在不久的将来能 开发出更加活跃和更有选择性的催化系统. 虽然仍有很 长的路要走, 但探索基于环糊精参与的过渡金属催化剂 合成的新方法、新技术以及催化新反应将前景光明.

\section{References}

[1] Lai, E.; Jean, M.; Shen, X. H. M. Supramolecular Chemistry: Concepts and Prospects, Peking University Press, Beijing, 2002 (in Chinese).

(莱恩, Jean, M., 沈兴海, 超分子化学: 概念和展望, 北京大学出 版社, 北京, 2002.)

[2] Xia, D. H.; Jiang, S. J.; Li, L.-L.; Xiang, Y. Z.; Zhu, L. J. Chin. J. 
Chem. Eng. 2016, 24, 146.

[3] Tong, L. H. M. Cyclodextrin Chemistry-Basics and Applications, Science Press, Beijing, 2001 (in Chinese). (童林荟, 环糊精化学一基础与应用, 科学出版社, 北京, 2001.)

[4] Shen, H. M.; Ji, H. B. Chin. J. Org. Chem. 2011, 32, 791 (in Chinese), (沈海民，纪红兵，有机化学, 2011, 32, 791.)

[5] Zhao, Y.; Huang, Y.; Zhu, H.; Zhu, Q.; Xia, Y. J. Am. Chem. Soc. 2016, $138,16645$.

[6] Menuel, S.; Léger, B.; Addad, A.; Monflier, E.; Hapiot, F. Green Chem. 2016, 18,5500

[7] Stewart, M. E.; Anderton, C. R.; Thompson, L. B.; Maria, J.; Gray, S. K.; Rogers, J.A.; Nuzzo, R. G. Chem. Rev. 2008, 108, 494.

[8] Xiao, J.; Qi, L. Nanoscale 2011, 3, 1383.

[9] Shanmugam, M.; Kim, K. J. Electroanal. Chem. 2016, 776, 82.

[10] Cravotto, G.; Gaudino, E. C.; Tagliapietra, S.; Carnaroglio, D.; Procopio, A. Green Proc. Synth. 2012, 1, 269.

[11] Hein, J. E.; Tripp, J. C.; Krasnova, L. B.; Sharpless, K. B.; Fokin, V. V. Angew. Chem., Int. Ed. 2009, 48, 8018.

[12] Dheer, D.; Rawal, R. K.; Singh, V.; Sangwan, P. L.; Das, P.; Shankar, R. Tetrahedron 2017, 73, 4295.

[13] Patil, R. N.; Vijay Kumar, A. ACS Omega 2017, 2, 6405.

[14] Messmer, E. Z. Phys. Chem. 1927, 126, 369.

[15] Kolb, H. C.; Finn, M. G.; Sharpless, K. B. Angew. Chem., Int. Ed. 2001, 113, 2056.

[16] Krasinski, A. ; Radic, Z.; Manetsch, R.; Raushel, J.; Taylor, P.; Sharpless, K. B.; Kolb, H. C. J. Am. Chem. Soc. 2004, 126, 12809.

[17] Hein, J. E.; Tripp, J. P.; Krasnova, L. B.; Sharpless, K. B.; Fokin, V. V. Angew. Chem., Int. Ed. 2009, 48, 1.

[18] Rostovtsev, V. V.; Green, L. G.; Fokin, V. V.; Sharpless, K. B. Angew. Chem., Int. Ed. 2002, 41, 2596.

[19] Tornoe, C. W.; Christensen, C.; Meldal, M. J. Org. Chem. 2002, 67, 3057.

[20] Aprahamian, I.; Dichtel, W. R.; Ikeda, T.; Heath, J. R.; Stoddart, J. F. Org. Lett. 2007, 9, 1287.

[21] Yigit, S.; Sanyal, R.; Sanyal, A. Chem. Asian J. 2011, 6, 2648.

[22] Yamada, Y. M. A.; Sarkar, S. M.; Uozumi, Y. J. Am. Chem. Soc. 2012, 134, 9285.

[23] Collinson, J.-M.; Wilton-Ely, J. D. E. T.; Diez-Gonzalez, S. Chem Commun. 2013, 49, 11358.

[24] Xiong, X.; Chen, H.; Tang, Z.; Jiang, Y. RSC Adv. 2014, 4, 9830.

[25] White, J. R.; Price, G. J.; Schiffers, S.; Raithby, P. R.; Plucinski, P. K.; Frost, C. G. Tetrahedron Lett. 2010, 51, 3913.

[26] Brotherton, W. S.; Michaels, H. A.; Simmons, J. T.; Clark, R. J.; Dalal, N. S.; Zhu, L. Org. Lett. 2009, 11, 4954.

[27] Hein, J. E.; Fokin, V. V. Chem. Soc. Rev. 2010, 39, 1302.

[28] Zhu, L.; Lynch, V. M.; Ansly, E. V. Tetrahedron 2004, 60, 7267.

[29] Zhang, H.; Tanimoto, H.; Morimoto, T.; Nishiyama, Y.; Kakiuchi, K. Tetrahedron 2014, 70, 9828.

[30] Ramesh, C.; Banerjee, J.; Pal, R.; Das, B. Adv. Synth. Catal. 2003, 345, 557.

[31] Sheng, S. R.; Wang, Q. Y.; Ding, Y.; Liu, X. L.; Cai, M. Z. Catal Lett. 2009, 128, 418

[32] Reddi, M. N. K.; Satheesh, K. B.; Anil, K. M.; Arulselvan, P.; Ibra- him, K. S.; Lasekan, O. Molecules 2012, 17, 7543.

[33] Ramesh, C.; Banerjee, J.; Pal, R.; Das, B. ChemInform 2010, 345, 557.

[34] Dabbawala, A. A.; Sudheesh, N.; Bajaj, H. C. Dalton. Trans. 2012 , 41, 2910.

[35] Datta, K. K. R.; Srinivasan, B.; Balaram, H.; Eswaramoorthy, M. J Chem. Sci. 2008, 120, 579 .

[36] Nie, R.; Sang, R.; Ma, X.; Zheng, Y.; Cheng, X.; Li, W.; Wu, Y. J. Catal. 2016, 344, 286

[37] Yao, Z.; Hong, S.; Zhang, W.; Liu, M.; Deng, W. Tetrahedron Lett. 2016, 57, 910.

[38] Zhang, P.; Meijide, S. J.; Driant, T.; Derat, E.; Zhang, Y.; Ménand, M. Angew. Chem. 2017, 129, 10961.

[39] (a) Kaboudin, B.; Abedi, Y.; Yokomatsu, T. Eur. J. Org. Chem. 2011, 6656 .

(b) Kaboudin, B.; Abedi, Y.; Yokomatsu, T. Org. Biomol. Chem. 2012, 10, 4543.

[40] Kaboudin, B.; Mostafalu, R.; Yokomatsu, T. ChemInform 2013, 44, 2262.

[41] Perez, A. L.; Moseguer, J. O.; Marques, P. R.; Corma, A. Angew. Chem., Int. Ed. 2013, 125, 11768

[42] Hoffmann, I.; Blumenröder, B.; Thumann,S. O. N.; Dommer, S.; Schatz, J. Green Chem. 2015, 17, 3844.

[43] Saito, N.; Taniguchi, T.; Hoshiya, N.; Shuto, S.; Arisawa, M.; Sato, Y. Green Chem. 2015, 17, 2358.

[44] (a) Zhong, R.; Pöthig, A.; Feng, Y.; Riener, K.; Herrmann, W. A.; Kühn, F. E. Green Chem. 2014, 16, 4955.

(b) Billingsley, K.; Buchwald, S. L. J. Am. Chem. Soc. 2007, 38, 3358 .

(c) Old, D. W.; Wolfe, J. P.; Buchwald, S. L. J. Am. Chem. Soc. 1999, 30, 4369 .

(d) Martin R.; Buchwald, S. L. Acc. Chem. Res. 2008, 41, 1461. (e) Vellakkaran, M.; Andappan, M. M. S.; Kommu, N. Green Chem. 2014, 16, 2788.

[45] Raihana, I. K.; Kasi, P. Green Chem. 2016, 18, 4791.

[46] Qi, M.; Tan, P. Z. Xue, F.; Malhi, H. S.; Zhang, Z. X.; Young, D. J. Rsc. Adv. 2014, 5, 3590.

[47] Zhou, X.; Guo, X.; Jian, F.; Wei, G. ACS Omega 2018, 3, 4418.

[48] Guo, Y.; Li, J.; Zhao, F.; Lan, G.; Li, L.; Liu, Y.; Yang, R. RSC Adv. 2016, 6,7950

[49] Imran, K. R.; Pitchumani, K. ACS Sustainable Chem. Eng, 2018.

[50] Poulos, T. L. Chem. Rev. 2014, 114, 3919.

[51] Sreenilayam, G.; Fasan, R. Chem. Commun. 2015, 51, 1532.

[52] Xu, X.; Li, C.; Tao, Z.; Pan, Y. Adv. Synth. Catal. 2015, 357, 3341.

[53] Wang, M. L.; Fang, G. D; Liu, P. Appl. Catal., B 2016, 188, 113.

[54] Guo, Y.; Guo, S.; Ren, J.; Zhai, Y.; Dong, S.; Wang, E. ACS Nano. 2010, 4, 4001 .

[55] Sharavath, V.; Sarkar, S.; Gandla, D.; Ghosh, S. Electrochim. Acta 2016, 210, 385.

[56] Mohamed, M. A.; Shukla, A.; Sandhya, K. Y. Environ. Prog. Sustainable 2016, 35, 1283.

[57] Subramanian, R.; Ponnusamy, V. J. Mater. Sci.: Mater. Electron. 2016, 28, 1 .

[58] Sun, N.; Wang, T.; Liu, C. Wood. Sci. Tenol. 2016, 50, 1. 